\title{
Acute pulmonary embolism
}

\author{
Author: Luke Howard ${ }^{\mathrm{A}}$
}

Pulmonary embolism, despite being common, often remains elusive as a diagnosis, and clinical suspicion needs to remain high when seeing a patient with cardiopulmonary symptoms. Once suspected, diagnosis is usually straightforward; however, optimal treatment can be difficult. Risk stratification with clinical scores, biomarkers and imaging helps to refine the best treatment strategy, but the position of thrombolysis in intermediate risk (submassive) pulmonary embolism remains a grey area. Pulmonary embolism response teams are on the increase to provide advice in such cases. Direct oral anticoagulants have been a major advance in treatment this decade, but are not appropriate for all patients. Follow-up of patients with pulmonary embolism should be mandatory to determine duration of anticoagulation and to assess for serious long-term complications.

KEYWORDS: Pulmonary embolism, thrombolysis

\section{Introduction}

Pulmonary embolism (PE) is a common presenting diagnosis in an emergency department. It may present with classical features such as breathlessness and pleuritic chest pain, but also less characteristically, for example insidious onset breathlessness over days-to-weeks or syncope ${ }^{1}$ with relatively few respiratory symptoms. Therefore, clinicians need to have a high degree of suspicion for PE in patients presenting with potential cardiopulmonary symptoms, since the consequences of missing or delaying the diagnosis of PE can be severe. As with most other areas of medicine, PE diagnosis and management has become heavily protocolised, ${ }^{2-5}$ but as will be discussed, there are still many grey areas in decision making in PE, necessitating experienced senior clinical decision making.

\section{Diagnosis}

Diagnostic algorithms and techniques have remained relatively unchanged over the past 10 years, with computed tomography (CT) pulmonary angiography (PA) being the principal tool (Fig 1). Since it involves ionising radiation, is not appropriate to use CTPA in all cases of suspected PE, hence clinical probability scores and

Author: ${ }^{\text {A }}$ Consultant pulmonologist, Hammersmith Hospital, London, UK

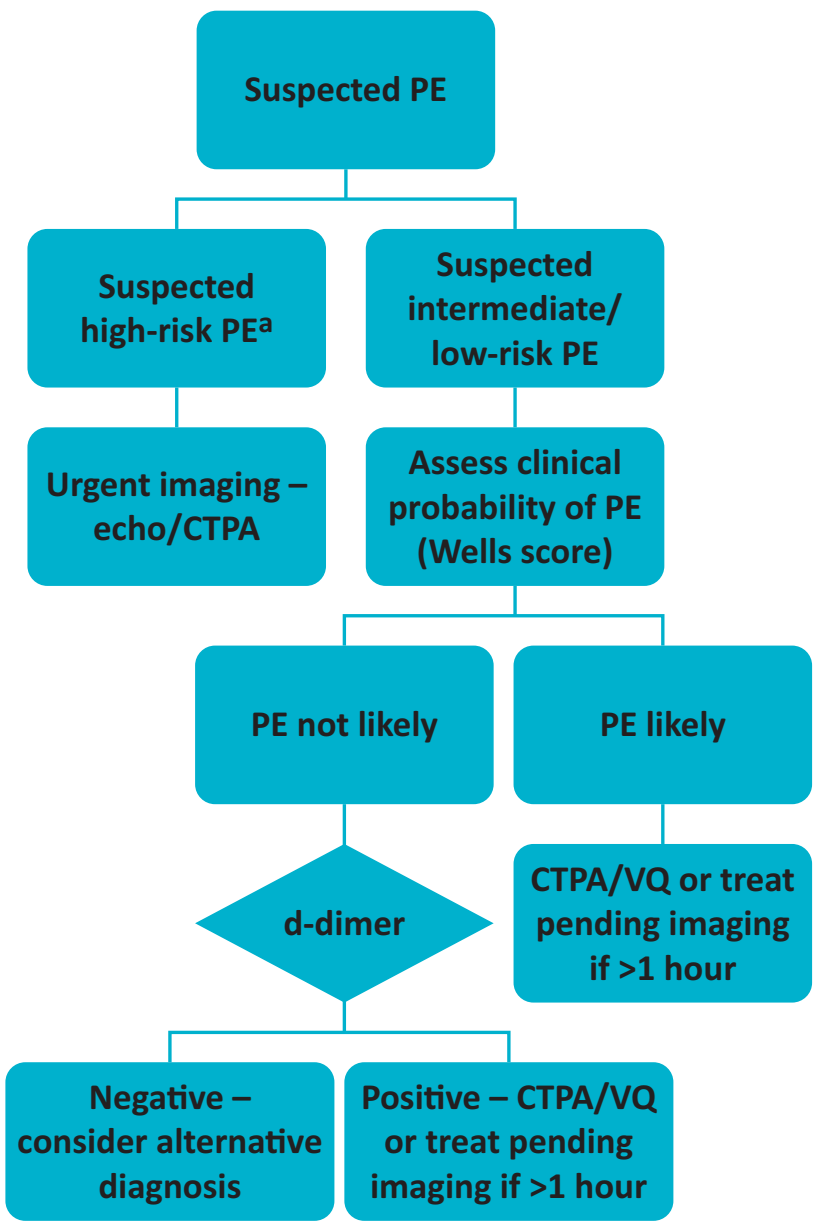

Fig 1. Diagnosis of pulmonary embolism (based on European Society of Cardiology guidelines and National Institute for Health and Care Excellence CG144). ${ }^{3,5}$ a Cardiac arrest; systolic blood pressure $<90 \mathrm{mmHg}$ or drop $\geq 40 \mathrm{mmHg}$ for 15 minutes not related to new-onset arrhythmia, hypovolaemia or sepsis. CTPA = computed tomography pulmonary angiography; $\mathrm{PE}=$ pulmonary embolism; $\mathrm{VQ}=$ ventilation perfusion.

d-dimer testing are used to filter out those with low probability of PE. While there are several clinical probability scores, the Wells score (Table 1) remains the predominant score in international guideline algorithms. Where clinical probability of PE is low, a normal d-dimer has a high negative predictive value for excluding PE, however where the d-dimer is elevated or the clinical probability of PE is high, diagnostic imaging should be performed. 


\begin{tabular}{|c|c|}
\hline Symptom & Score \\
\hline $\begin{array}{l}\text { Clinical signs and symptoms of DVT (minimum of } \\
\text { leg swelling and pain with palpation of the deep } \\
\text { veins) }\end{array}$ & 3 \\
\hline An alternative diagnosis is less likely than PE & 3 \\
\hline Heart rate $>100$ beats/minute & 1.5 \\
\hline Immobilisation or surgery in the previous 4 weeks & 1.5 \\
\hline Previous DVT/PE & 1.5 \\
\hline Haemoptysis & 1 \\
\hline $\begin{array}{l}\text { Malignancy (on treatment, treated in the last } \\
6 \text { months, or palliative) }\end{array}$ & 1 \\
\hline Clinical probability & Total score \\
\hline PE unlikely & $\leq 4$ \\
\hline PE likely & $>4$ \\
\hline
\end{tabular}

Interest is increasing in using age-adjusted d-dimer, however, evidence has not yet reached significance to be considered part of routine practice. In certain circumstances, in particular pregnancy, d-dimer, inflammation and cancer cannot be used to triage patients for imaging.

An alternative strategy to $\mathrm{CT}$ is to consider ultrasound Doppler of the leg veins where it is important to avoid ionising radiation, such as in the pregnant patient, but this strategy in the nonpregnant patient is not of sufficient yield to justify its use, resulting in a positive finding in approximately 1 in 10 cases. $^{6}$ Ventilationperfusion (VQ) scanning can be used where available, in the presence of a normal chest radiograph. Performing a half dose perfusion scan is also an option in the pregnant patient.

With advancing technology, CTPA is able to detect smaller filling defects in the pulmonary circulation. Where it is certain that these filling defects are genuine pulmonary emboli, it is important for the clinician to assess whether such small volume clots account for the presentation, for example, is an isolated subsegmental PE sufficient to account for patient presenting with syncope? Conversely, where there has been poor contrast opacification or breathing artefact, scans may be misinterpreted as showing small peripheral emboli and there is poor inter-observer agreement where emboli are $<6 \mathrm{~mm}$ or subsegmental. ${ }^{7}$ Given the important consequences of diagnosing PE, such as the need for lifelong anticoagulation, it is important in these circumstances to review scans carefully and sometimes consider repeating or opting for a different modality.

\section{Risk stratification}

Risk stratification plays an important role in management of the suspected and confirmed PE (Fig 1). Systemic lysis is indicated for the high-risk (previously known as massive) PE or in the context of cardiac arrest due to suspected PE. Where lysis is contraindicated, surgical embolectomy should be considered. This group represents a relatively small proportion of patients presenting with PE $(<5 \%)$ and the greater challenge is to identify patients in whom

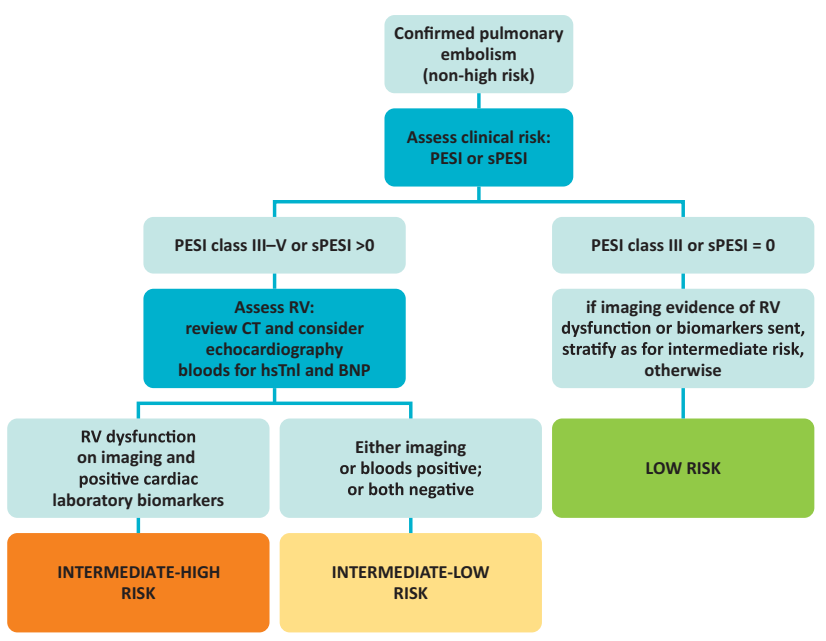

Fig 2. Risk stratification of non-high risk pulmonary embolism (based on European Society of Cardiology 2014 guidelines). ${ }^{3}$ BNP = brain natriuretic peptide; $\mathrm{CT}$ = computed tomography; hs $\mathrm{TnI}=$ high sensitive Troponin-I; PESI = Pulmonary Embolism Severity Index; RV = right ventricle; sPESI = simplified Pulmonary Embolism Severity Index .

thrombolysis/reperfusion therapy should be considered from the non-high-risk group.

The European Society of Cardiology (ESC) 2014 guidelines pioneered the concept of risk-based management (Fig 2). ${ }^{3}$ Many clinical risk scores exist, but perhaps the one that has gained the most traction is the Pulmonary Embolism Severity Index (PESI) and subsequently its simplified version (sPESI; Table 2). These scores can be made at the bedside using clinical history and simple observations. Intermediate risk patients can be considered those in PESI classes III-V or SPESI $>0$. This represents a large cohort of patients and most will not benefit from thrombolysis and therefore further risk stratification is required.

The ESC guidelines suggest that intermediate risk patients who also have an elevated cardiac biomarker, such as troponin or $\mathrm{N}$-terminal pro-brain natriuretic peptide, and have evidence of right ventricular dysfunction on CT or echocardiography, should be labelled as intermediate-high risk and be considered for thrombolysis/reperfusion. However, the Pulmonary Embolism International Thrombolysis Trial (PEITHO), a large study of over 1,000 patients with this risk profile, demonstrated no mortality benefit at 7 or 30 days with thrombolysis, but did demonstrate a significant increase in adverse events related to bleeding. ${ }^{8}$ In the extension study, long-term outcomes including incidence of chronic thromboembolic pulmonary hypertension were no different. ${ }^{9}$

When comparing against the expected mortality from the PESI scoring system (Table 2), the overall risk of mortality was low in the short-term PEITHO study, approximately $2 \%$, which may reflect either better overall patient management as part of a clinical trial, such as closer observation, or a bias towards excluding enrolment of higher-risk patients according to clinician judgement. Therefore, there does remain a need to identify a further subgroup of intermediate-high risk patients who might benefit from more aggressive therapy. One study has suggested that patients with a combination of a sPESI greater than zero, positive brain natriuretic peptide and troponin, and finding of proximal deep vein thrombosis have the highest risk of complicated outcomes, with positive cardiac biomarkers outperforming, and therefore negating 
Table 2. Pulmonary Embolism Severity Index and simplified Pulmonary Embolism Severity Index

PESI

sPESI

Demographics

Age in years, $n$

Sex $=$ male

$+10$

1 point (if age $>80$ years)

Comorbidities

Cancer

$+30$

$+10$

Heart failure

$+10$

Chronic lung disease

Clinical findings

Pulse $\geq 110 \mathrm{bpm}$

$+20$

SBP $<100 \mathrm{mmHg}$

$+30$

$\mathrm{RR} \geq 30$ breaths/min

Temperature $<36^{\circ} \mathrm{C}$

$+20$

$+20$

$+60$

$+20$

Total PESI points

$\leq 65$

66-85

86-105

106-125

$\geq 126$

Total sPESI points

0

$>0$
0 points

1 point

1 point

1 point

1 point

0 points

0 points

0 points

1 point

PESI 30-day mortality

$0-1.6 \%$

$1.7-3.5 \%$

$3.2-7.1 \%$

$4.0-11.4 \%$

$10.0-24.5 \%$

sPESI 30-day mortality

$1.0 \% ; 95 \%$ CI $0.0-2.1 \%$

$10.9 \% ; 95 \%$ CI $8.5-13.2 \%$
High

= systolic blood pressure; sPESI =

$\mathrm{CI}=$ confidence interval; $\mathrm{PESI}=$ Pulmonary Emboli simplified Pulmonary Embolism Severity Index.

the need for, right ventricular imaging on multivariate analysis. ${ }^{10}$ A post-hoc analysis of the PEITHO study (ie those already in the intermediate-high risk group) suggested that two or more of the following: systolic blood pressure $\leq 110 \mathrm{mmHg}$, respiratory rate $>20$ breaths per minute, history of heart failure and active cancer were associated with the highest risk of major adverse outcomes. ${ }^{11}$ Thus, there appears to be a number of risk factors associated with unfavourable outcomes but as yet no clear guidance can be given on when to administer thrombolysis/reperfusion.

Of course, not only PE-related mortality needs to be considered, but also the risk of major bleeding when contemplating thrombolysis. The most important risk factor is age, with patients over the age of 65 being the highest risk of major bleeding. ${ }^{12}$ Balancing the risk of bleeding against risk of PE-related mortality requires shared decision making between senior clinicians and patient, including informed consent. In recent years, there has been a rise of pulmonary embolism response teams (PERTs), particularly in the USA but more recently in the UK, to provide expert advice in such scenarios. ${ }^{13}$ The need for PERTs has been increased with the advent of more complex therapies such as catheter directed thrombolysis, which uses very low doses of lytic, ${ }^{14}$ as well as the potential use of half-dose systemic lysis. ${ }^{15} \mathrm{~A}$ recent network meta- analysis has suggested that catheter directed lysis and half-dose lysis may provide optimal outcomes in terms of reduced mortality and bleeding, but the supporting data are relatively weak. ${ }^{16}$ Most recently, the $\operatorname{EKOS}^{\circledR}$ system, which co-ministers local thrombolysis with ultrasound to increase fibre in separation via pulmonary artery catheter, has been shown to improve right ventricular to left ventricular diameter ratios with low doses of tissue plasminogen activator. ${ }^{14}$ The role for this particular technique and half-dose thrombolysis are yet to find their place in guideline algorithms, and their use remains at the discretion of senior clinicians / PERTs.

Patients with intermediate-low risk PE should usually be admitted to hospital for a short inpatient stay for anticoagulation treatment. There are no clear guidelines on how long patients should be admitted, but 48 hours seems appropriate.

Patients with low risk PE can now be considered for full outpatient management following the British Thoracic Society guidelines (Figure 3). Patients in PESI I/II or sPESI 0 may be sent home provided they do not have any other social or medical reasons for admission (Box 1). Alternatively another set of criteria may be used, known as the Hestia criteria, which have been specifically validated outpatient selection. ${ }^{17}$ Clinicians may wish to use a negative cardiac biomarker to 'override' the finding of right ventricular dilatation 


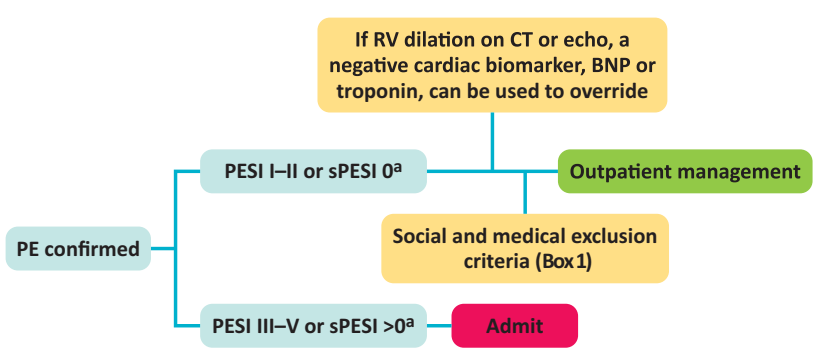

Fig 3. British Thoracic Society algorithm for selection of patients for outpatient management. ${ }^{4 a}$ Alternatively, Hestia criteria may be used without subsequent need for additional social/medical criteria. BNP = brain natriuretic peptide; $\mathrm{CT}=$ computed tomography; $\mathrm{PESI}=$ Pulmonary Embolism Severity Index; RV = right ventricle; $\mathrm{SPESI}=$ simplified Pulmonary Embolism Severity Index.

on CT. When managing patients via an ambulatory pathway, it is of utmost importance to provide patients with good information, advice on when to re-present to the emergency department, follow up closely after discharge and arrange further tests, where required, to investigate, such as cancer.

\section{Anticoagulation}

The mainstay of treatment of PE is anticoagulation. Until recently, the standard of care was low molecular weight heparin (LMWH) followed by warfarin, but in recent years this has been replaced by the direct oral anticoagulants (DOACs). Apixaban, dabigatran, edoxaban and rivaroxaban have all been licensed for the treatment of venous thromboembolism. ${ }^{18}$ Dabigatran and edoxaban both require a minimum 5 -day lead-in period with $\mathrm{LMWH}$, whereas apixaban and rivaroxaban may be administered as soon as PE is confirmed with an initial high-dose regime tapering down at 7 and 21 days, respectively. Overall, the DOACs as a class are non-inferior to LMWH/warfarin in terms of efficacy and appear to have lower

Box 1. British Thoracic Society exclusion criteria for outpatient management

Haemodynamic instability (HR $>110$ bpm, SBP $<100 \mathrm{mmHg}$, requirement for inotropes or critical care, requirement for thrombolysis or embolectomy)

Oxygen saturations $<90 \%$ on air

Active bleeding or risk of major bleeding (eg recent GI bleed or surgery, previous intracranial bleeding, uncontrolled hypertension)

On full-dose anticoagulation at the time of the PE

Severe pain (for example requiring intravenous analgaesia)

Chronic kidney disease stage 4 or 5 (eGFR $<30 \mathrm{~mL} / \mathrm{min}$ ) or severe liver disease

Heparin-induced thrombocytopaenia within the last year and where there is no alternative to repeating heparin treatment

Other medical or social reasons necessitating initial inpatient management

eGFR, estimated glomerular filtration rate; GI = gastrointestinal; $H R$, heart rate; $\mathrm{PE}=$ pulmonary embolism; SBP, systolic blood pressure. risk of bleeding. ${ }^{18}$ Detailed discussion of the use of DOACs in specific circumstances is beyond the scope of this article. Where anticoagulation cannot safely be administered due to high risk of bleeding, inferior vena cava filters may be used, but it is important to remove these as soon as anticoagulation can be administered because of the risk of long-term complications. There are no other routine indications for use of inferior vena cava filters. ${ }^{19}$

\section{Special circumstances}

\section{Pregnancy}

PE in pregnancy should be managed in the same way as in patients who are not pregnant but with several caveats: diagnosis is discussed above; the PESI scoring system cannot be applied and thus a decision as to whether to manage a patient as an outpatient can only be made after discussion between a senior physician and obstetrician; patients should be anticoagulated with LMWH, not DOACs; and pregnancy should be considered a relative contraindication to thrombolysis.

\section{Cancer}

Guidelines presently recommend the use of LMWH as the first line anticoagulant in cancer-associated thrombosis (CAT), however, two recent studies with edoxaban and rovaroxaban have shown improved efficacy over LMWH in CAT, but increased bleeding. The International Society of Thrombosis and Haemstosasis, have produced guidance to state that these two DOACs may be considered in place of $\mathrm{LMWH}$ where bleeding risk is low. ${ }^{20}$

PE may be the presenting feature of underlying malignancy, but screening for cancer remains controversial. At present the National Institute for Health and Care Excellence (NICE) guidelines (CG144) still recommend offering patients with first unprovoked PE over the age of 40 investigation for cancer including physical examination, blood tests (including full blood count, calcium and liver function tests), urinalysis, abdomino-pelvic CT and mammogram in women. ${ }^{5}$

\section{Right heart thrombus}

The presence of right heart thrombus increases the risk of mortality due to the risk of pulmonary outflow tract obstruction and increased right ventricle afterload. ${ }^{21}$ Free-floating clot in the right-sided cardiac chambers should be considered an indication for systemic thrombolysis, however if the clot is straddling a patent foramen ovale, then surgical embolectomy may be appropriate. Anticoagulation is preferred for patients with a clot adherent to the endocardium unless it is mobile and risks valvular obstruction, in which case surgery may also be considered. ${ }^{22}$

\section{Follow-up}

Detailed discussion regarding follow-up of patients with PE is beyond the scope of this review, however it is critical that patients should be fed in to a clinic to review the outcome of treatment, in particular to assess for the possibility of chronic thromboembolic pulmonary hypertension and determine duration of anticoagulation. Where investigations for possible underlying malignancy have been performed, this also provides an additional safety net. There is also a high burden of psychological issues following PE, which can largely be addressed by a physician, ${ }^{23}$ but also referred on for psychological support where necessary. 


\section{References}

1 Prandoni P, Lensing AW, Prins MH et al. Prevalence of pulmonary embolism among patients hospitalized for syncope. N Engl J Med 2016;375:1524-31.

2 Kearon C, Akl EA, Ornelas ] et al. Antithrombotic therapy for VTE disease: CHEST guideline and expert panel report. Chest 2016;149:315-52.

3 Konstantinides SV, Torbicki A, Agnelli G et al. 2014 ESC guidelines on the diagnosis and management of acute pulmonary embolism. Eur Heart J 2014;35:3033-69k.

4 Howard L, Barden S, Condliffe R et al. British Thoracic Society guideline for the initial outpatient management of pulmonary embolism (PE). Thorax 2018;73(Suppl 2):ii1-29.

5 National Institute for Health and Care Excellence. Venous thromboembolic diseases: the management of venous thromboembolic diseases and the role of thrombophilia testing. Clinical guideline [CG144]. London: NICE, 2015.

6 Righini M, Le Gal G, Aujesky D et al. Diagnosis of pulmonary embolism by multidetector $\mathrm{CT}$ alone or combined with venous ultrasonography of the leg: a randomised non-inferiority trial. Lancet 2008;371:1343-52.

7 Miller WTJr, Marinari LA, Barbosa EJr et al. Small pulmonary artery defects are not reliable indicators of pulmonary embolism. Ann Am Thorac Soc 2015;12:1022-9.

8 Meyer G, Vicaut E, Danays T et al. Fibrinolysis for patients with intermediate-risk pulmonary embolism. $N$ Engl J Med 2014;370:1402-11.

9 Konstantinides SV, Vicaut E, Danays T et al. Impact of thrombolytic therapy on the long-term outcome of intermediate-risk pulmonary embolism. J Am Coll Cardiol 2017;69:1536-44.

10 Jimenez D, Kopecna D, Tapson V et al. Derivation and validation of multimarker prognostication for normotensive patients with acute symptomatic pulmonary embolism. Am J Respir Crit Care Med 2014;189:718-26.

11 Barco S, Vicaut E, Klok FA et al. Improved identification of thrombolysis candidates amongst intermediate-risk pulmonary embolism patients: implications for future trials. Eur Respir ] 2018;51:1701775.

12 Chatterjee S, Chakraborty A, Weinberg I et al. Thrombolysis for pulmonary embolism and risk of all-cause mortality, major bleeding, and intracranial hemorrhage: a meta-analysis. JAMA 2014;311:2414-21.

13 Dudzinski DM, Piazza G. Multidisciplinary pulmonary embolism response teams. Circulation 2016;133:98-103.
14 Tapson VF, Sterling K, Jones N et al. A randomized trial of the optimum duration of acoustic pulse thrombolysis procedure in acute intermediate-risk pulmonary embolism: The OPTALYSE PE trial. JACC Cardiovasc Interv 2018;11:1401-10.

15 Wang C, Zhai Z, Yang Y et al. Efficacy and safety of low dose recombinant tissue-type plasminogen activator for the treatment of acute pulmonary thromboembolism: a randomized, multicenter controlled trial. Chest 2010;137:254-62.

16 Jimenez D, Martin-Saborido C, Muriel A et al. Efficacy and safety outcomes of recanalisation procedures in patients with acute symptomatic pulmonary embolism: systematic review and network meta-analysis. Thorax 2018;73:464-71.

17 Zondag W, Vingerhoets LM, Durian MF et al. Hestia criteria can safely select patients with pulmonary embolism for outpatient treatment irrespective of right ventricular function. J Thromb Haemost 2013;11:686-92.

18 van der Hulle T, Huisman MV, Klok FA. Meta-analysis of the efficacy and safety of new oral anticoagulants in patients with cancer-associated acute venous thromboembolism: reply. J Thromb Haemost 2014;12:2138-9.

19 Mismetti P, Laporte S, Pellerin O et al. Effect of a retrievable inferior vena cava filter plus anticoagulation vs anticoagulation alone on risk of recurrent pulmonary embolism: a randomized clinical trial. JAMA 2015;313:1627-35.

20 Khorana AA, Noble S, Lee AYY et al. Role of direct oral anticoagulants in the treatment of cancer-associated venous thromboembolism: guidance from the SSC of the ISTH. J Thromb Haemost 2018;16:1891-94.

21 Koc M, Kostrubiec M, Elikowski W et al. Outcome of patients with right heart thrombi: the Right Heart Thrombi European Registry. Eur Respir ] 2016;47:869-75.

22 Condliffe R, Elliot CA, Hughes RJ et al. Management dilemmas in acute pulmonary embolism. Thorax 2014;69:174-80.

23 Hunter R, Lewis S, Noble S et al. 'Post-thrombotic panic syndrome': A thematic analysis of the experience of venous thromboembolism. Br J Health Psychol 2017;22:8-25.

Address for correspondence: Dr Luke Howard, consultant pulmonologist, National Pulmonary Hypertension Service, Dept of Cardiology, Hammersmith Hospital, Imperial College Healthcare NHS Trust, Du Cane Road, London W12 OHS, UK. Email: I.howard@imperial.ac.uk 